\title{
An evolutionary algorithm for inverse RNA folding inspired by Lévy flights
}

\author{
Nono S. C. Merleau ${ }^{\mathrm{a}}$ and Matteo Smerlak ${ }^{\mathrm{a}}$ \\ ${ }^{\mathrm{a}}$ Max Planck Institute for Mathematics in the Sciences, Leipzig, Germany
}

\begin{abstract}
A Lévy flight is a random walk with step sizes that follow a heavy-tailed probability distribution. This type of random walk, with many small steps and a few large ones, has inspired many applications in genetic programming and evolutionary algorithms in recent years, but is yet to be applied to RNA design. Here we study the inverse folding problem for RNA, viz. the discovery of sequences that fold into given target secondary structures. We implement a Lévy mutation scheme in an updated version of aRNAque, an evolutionary inverse folding algorithm, and apply it to the design of RNAs with and without pseudoknots. We find that the Lévy mutation scheme increases the diversity of designed RNA sequences and reduces the average number of evaluations of the evolutionary algorithm. The results show improved performance on both Pseudobase++ and the Eterna100 datasets, outperforming existing inverse folding tools. We propose that a Lévy flight offers a better standard mutation scheme for optimizing RNA design.
\end{abstract}

\section{Introduction}

The function of non-coding RNA, which includes gene expression regulation (miRNAs, piRNAs, lncRNAs), RNA maturation (snR- NAs, snoRNAs) and protein synthesis (rRNAs, tRNAs), strongly depends on the hierarchical folding of RNA molecules. Given their sequence of bases (primary structure), RNAs fold into secondary structures, such as stem loops and pseudoknots, before folding into higher level (tertiary and quaternary) structures. The secondary structure can be considered as a list of base pairs, including canonical, Watson-Crick pairs $(1,2)$; non-canonical interactions, that occur with reduced frequency; and crossing or pseudoknot interactions (when two canonical or non-canonical interactions cross each other) (3).

Here, we consider the RNA secondary structure inverse folding problem. The goal is to find RNA sequences that fold into a given target secondary structure, with or without pseudoknots. Considering pseudoknots in designing functional RNAs is vital given their role in realising biological functions. In modern bio-engineering, one must solve the RNA inverse folding problem to be able to design RNA molecules performing specific functions (4-6).

A key prerequisite to addressing the RNA inverse problem is a reliable solution to the folding problem. Computationally folding an RNA molecule consists of searching in the space of all possible secondary structures for one that minimises the free energy. Designing sequences for a pseudoknotted target structure is computationally more expensive than a pseudoknot-free target because of the complexity of the folding algorithms required. Specifically, the time complexity of the pseudoknot-free secondary structure prediction is $O\left(n^{3}\right)$ when using dynamic programming approaches such as RNAfold (7), or less with heuristic folding methods (e.g. $O(n)$ for LinearFold and $O\left(n^{2} \log n\right)$ for RAFFT (8)). By contrast, when considering a special class of pseudoknots, the time complexity of folding goes up to $O\left(n^{6}\right)$ for an exact thermodynamic prediction using a dynamic programming approach such as (9). In this work, we consider only two heuristics tools (IPknot (10) and HotKnots (11)) chosen for their lower time complexity $O\left(n^{4}\right)$.

Many of the studies addressing the inverse folding of RNA considered only pseudoknot-free secondary structures (1219). There are, however, three exceptions, the most recent of which is antaRNA (20) utilising the "ant-colony" optimisation technique. The technique begins with an initial sequence generated via a weighted random search; next the solutions are evaluated, and the sequence fitness values are used to refine the weights and improve the sequences over generations. Another approach (Modena) implements a multi-objective evolutionary algorithm measuring both the stability of the designed sequences and the similarities of folded sequences to the target structure. Although the first version of Modena was implemented for pseudoknot-free structure (15), it has since been extended to support pseudoknotted RNAs and a new crossover operator (21). Inv (22) is the first inverse folding tool handling pseudoknotted RNA target structures, but is restricted to a specific type of pseudoknot pattern called 3 -crossing nonplanar pseudoknots. In a prior publication (23), we presented aRNAque, a simple evolutionary inverse folding algorithm guided by local (or one-point mutations). Although a local search can efficiently discover optima in a simple landscape, more complex landscapes pose challenges to the design of evolutionary algorithms that rely solely on local search. This is especially true on a neutral landscape where local search may be inefficient or risk getting stuck on a plateau (or local optimum). To avoid this pitfall, we propose here an extension of aRNAque which implements a new mutation scheme inspired by Lévy flights (called Lévy mutation) and supports pseudoknotted RNA target structures.

Lévy flights are random walks with a heavy-tailed step size distribution. The concept originates in the work of Mandelbrot on the fluctuation of commodities prices in the $1960 \mathrm{~s}$ (24), but has since found many more physical applications (25). Lévy flights also play a key role in the context of animal foraging, perhaps because they provide an optimal balance between exploration and exploitation $(26,27)$. For a 
$(\mathrm{A})$

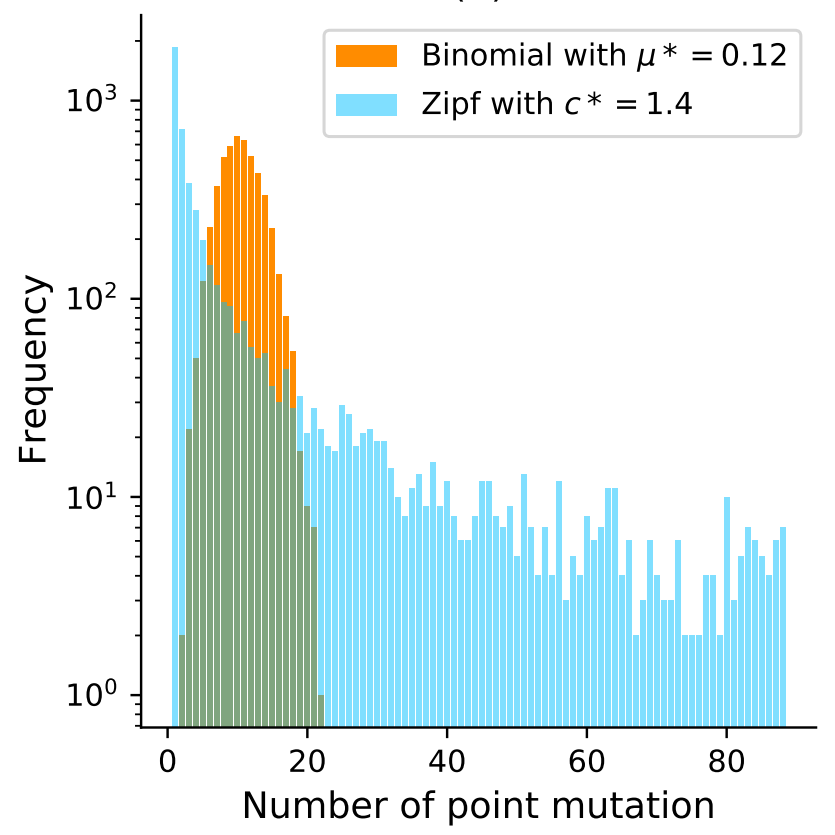

(B)

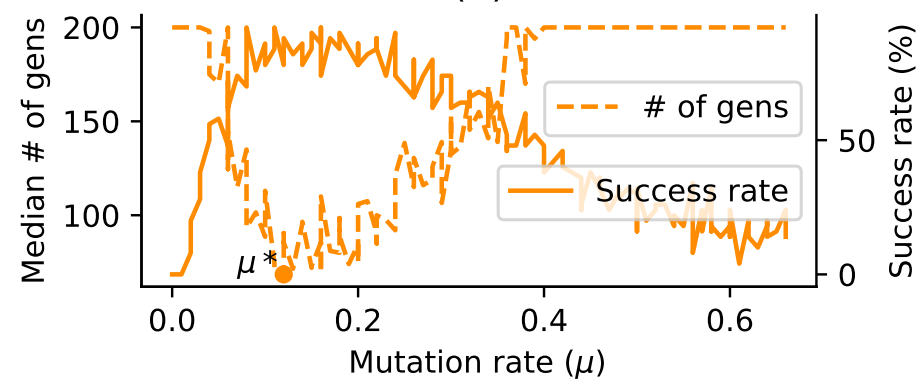

(C)

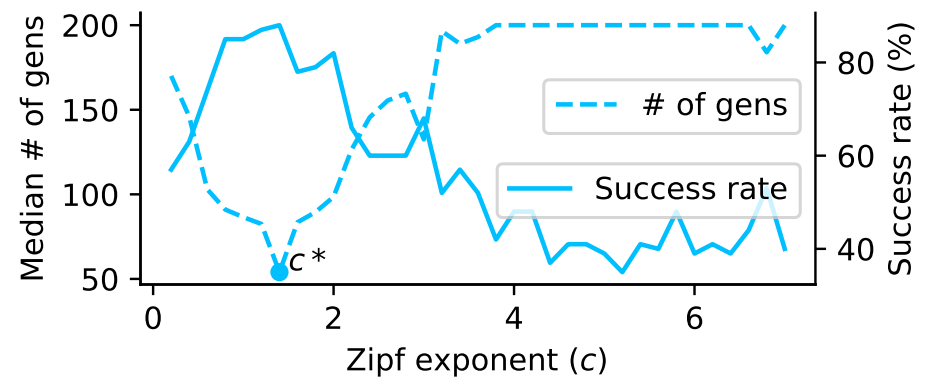

Fig. 1. (A) Samplings Binomial and Zipf distributions for the best binomial mutation rate $\mu^{*}$ (respectively $c^{*}$ for the best Zipf exponent parameter). Both distributions have a mean of 8.7 point mutations for a sequence of length 88 nucleotides. (B) Tuning of binomial mutation rate parameter. For each $\mu \in[0,1]$ with a step size of 0.005 and the pseudoknotted target PKB0 0342 of length 88, 50 sequences were designed using aRNAque. (B) shows the median generations and the success percentage vs. the mutation rate $(\mu)$. The best mutation rate is $\mu^{*}=0.085$ (with a median number of generation 93.5 and a success rate of $92 \%$ ). (C) Tuning of Levy exponent. Similar to $(B)$, for each $c \in[0,7]$ with a step size of 0.1 and for the same pseudoknotted target structure, 100 sequences were designed using aRNAque. It shows the median generations and the percentage of success vs. the exponent parameter $(c)$. The Zipf exponent distribution that produced the highest success rate and the minimum number of generations is $c^{*}=1.4$.

recent review of applications of Lévy flights in biology from the molecular to the ecological scale (28).

Similar to a Lévy flight, a Lévy mutation scheme allows simultaneous search at all scales over the landscape. New mutations most often produce nearby sequences (one-point mutations), but occasionally generate mutant sequences which are far away in genotype space (macro-mutations). In this work, the distribution of the number of point mutations at every step is taken to follow a Zipf distribution (29).

The optimization approach implemented in aRNAque is an evolutionary algorithm, which consists of a population of RNA sequences that all perform separate random walks (are mutated) in the space of possible sequences, and whose stepsizes (number of point mutations) follow a Zipf distribution. After each step, the probability of surviving is proportional to the fitness of each sequence, which is evaluated by its ability to approximate a given target structure. We provide a brief overview of that approach in the following subsection.

Earlier works have applied similar ideas in genetic programming (30), and in differential evolutionary algorithms (31). This has motivated us to investigate the possible benefit of a Lévy flight in the design of RNA sequences. Using a Lévy mutation scheme, we aim to speed up our prior evolutionary algorithm and increase the diversity of the designed RNA sequences.

We compared the performance of our newly modified version of aRNAque to existing tools through a benchmark on two well-known RNA datasets: PseudoBase+++ (32) for the pseudoknotted targets and Eternalo0 (33) for the pseudoknot-free targets. On the PseudoBase +++ dataset, the difference between the local mutation and the Lévy mutation with respect to the number of generations (or evaluations) was significant (with a $p$-value $\approx 0.00004$ ). Using the two pseudoknot folding tools HotKnots and IPknot, our designed sequences were of better quality than the ones produced by antaRNA regarding the average base pair distance to the desired targets. We performed a second benchmark on the Eterna100 dataset. Considering the Eterna100-V1 dataset, the Lévy mutation scheme solved 89 targets out of 100 whereas the local mutation scheme solved 91/100. To compare aRNAque to the existing pseudoknot-free inverse folding tools, we combined the two benchmark results obtained using both mutation schemes, and we counted the total number of distinct targets solved. aRNAque designs successfully $92 / 100$ of the Eterna100-V1 dataset and 94/100 of the Eterna100-V2 dataset.

\section{Evolutionary algorithm (EA)}

Below, we provide a brief overview of our evolutionary search algorithm and our mutation scheme.

Overview. In general, an evolutionary search algorithm on any fitness landscape consists of three main parts, which in the context of RNA inverse folding are as follows:

- Initialization: generating a random initial population of RNA sequences compatible with the given target secondary structure. 

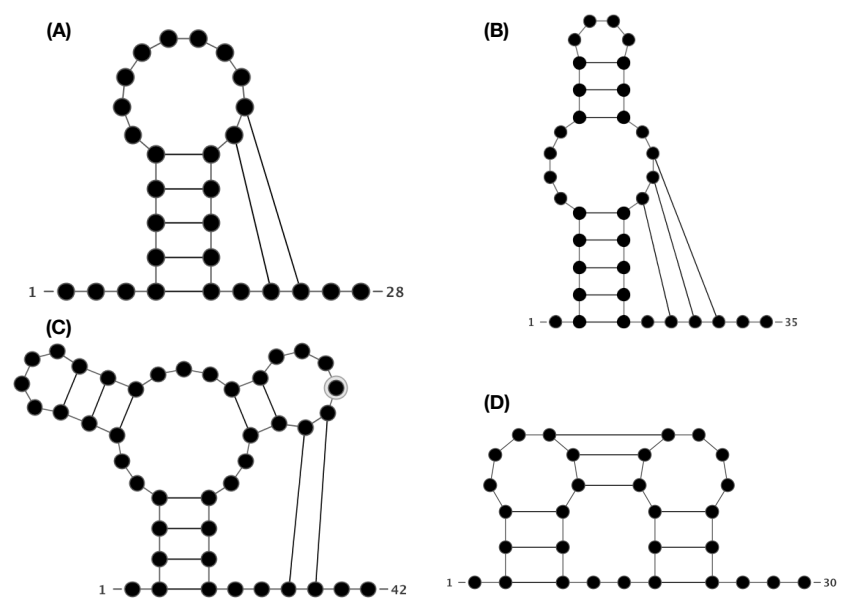

Fig. 2. Types of pseudoknots accommodated by aRNAque. (A) Hairpin (H-type) pseudoknot. (B) Bulge (B-type) pseudoknot. (C) Complex hairpin (cH-type) pseudoknot. (D) Kissing hairpin (K-type) pseudoknot.

- Evaluation and selection: evaluating a population of RNA sequences consists of two steps: 1 ) fold each sequence into a secondary structure and assign it a weight based on its similarity to the target structure. 2) select a weighted random sample with replacement from the current population to generate a new population. A detailed description of the objective function used in aRNAque is provided in (23).

- Mutation (or move) operation: define a set of rules or steps used to produce new sequences from the selected or initial ones. This component is elaborated further in the next subsection.

Mutation mode. For a given target RNA secondary structure $\sigma *$ of length $L$, the space of potential solutions to the inverse folding problem is $S=\{A, C, G, U\}^{L}$. An evolutionary algorithm explores the space $S$ through its move (or mutation) operator.

Given a sequence $\phi \in S$, a sequence $\phi^{\prime} \in S$ is said to be an $n$-point mutation of $\phi$ if it differs from $\phi$ at $n$ nucleotides; i.e. $h\left(\phi, \phi^{\prime}\right)=n$ where $h(.,$.$) is the hamming distance on S$.

A mutation mode is a random variable $U$ taking values in $\{1, \ldots, L\} . P(U=n)$ is defined as the probability that, exactly $n$ nucleotides, selected uniformly at random undergo point mutation during a mutation event. $U$ can generally be any probability distribution. We examined the binomial and Zipf distributions for local and Levy search, respectively:

- Binomial mutation: $U$ has a binomial distribution:

$$
P(U=n)=\left(\begin{array}{l}
l \\
n
\end{array}\right) \mu^{n}(1-\mu)^{l-n}
$$

for some $0 \leq \mu \leq 1$, such that $u=\mu \cdot l$. We can think of this mutation mode arising from each nucleotide of an RNA sequence independently undergoing a point mutation with probability $\mu$, i.e. $\mu$ is the per-nucleotide or point mutation rate.
- Lévy mutation: $U$ has a Zipf distribution given by:

$$
P(U=n)=\frac{1 / n^{c}}{\sum_{k=1}^{l} 1 / k^{c}}
$$

where $c>0$ is the value of the exponent characterizing the distribution. Larger values of $c$ are associated a greater proportion of local search, while smaller values of $c$ imply a greater proportion of long-range search.

Figure 1 shows the distribution of the number of point mutations on a sequence of length 88 nucleotides for both mutation schemes. Both distributions have the same mean, but differ markedly in their tails.

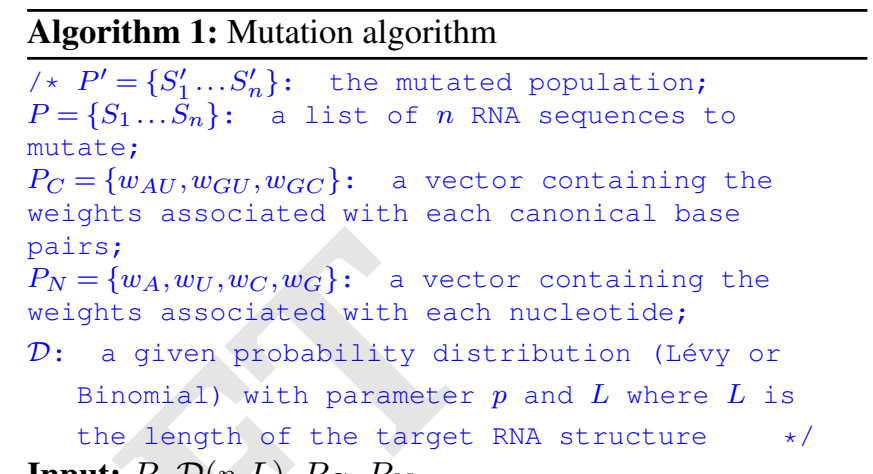

Input: $P, \mathcal{D}(p, L), P_{C}, P_{N}$

Output: $P^{\prime}$

$1\left\{B_{i}\right\} \sim \mathcal{D}(p, L)$, where $i \in\{1,2, \ldots, n\} ; \quad / /$ Draw $n$ random numbers that follows a given distribution $\mathcal{D}(p, L)$ (Lévy or Binomial). $\quad B_{i}$ is the number base pairs to mutate

$2\left\{U_{i}\right\} \sim \mathcal{D}(p, L)$, where $i \in\{1,2, \ldots, n\}$; // Draw $n$ random numbers that follows the same distribution as $B_{i}$ (Lévy or Binomial). $U_{i}$ is the number non base pair positions to mutate 3 for $i \in\{1,2, \ldots, n\}$ do

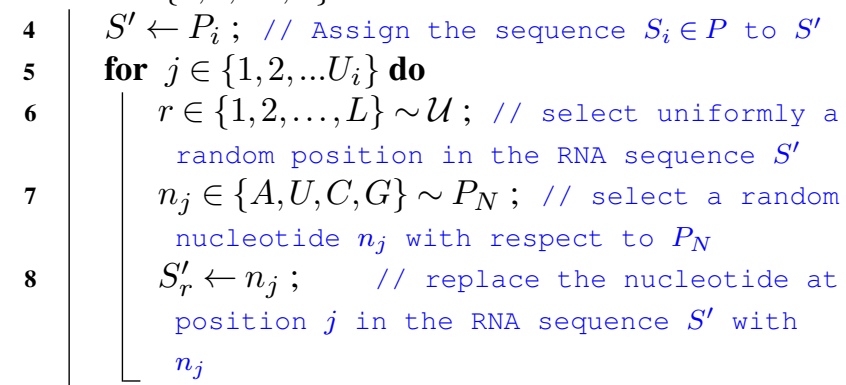
9 for $j \in\left\{1,2, \ldots B_{i}\right\}$ do

$$
\begin{aligned}
& \quad k_{j} \in\{A U, U A, C G, G C, G U, U G\} \sim P_{C} ; \\
& \quad / / \text { select a random base pair } k_{i} \text { with } \\
& \text { respect to } P_{C} \\
& b \in\left\{\left(b_{1}, b_{2}\right)_{i}\right\} \sim \mathcal{U} ; \text { // select uniformly a } \\
& \text { random pair of base pair positions } \\
& S_{b}^{\prime} \leftarrow k_{j} ; \quad / / \text { replace respectively the } \\
& \text { nucleotides at the base pair position } \\
& b_{i} \in b \text { by } k \in k_{j}
\end{aligned}
$$



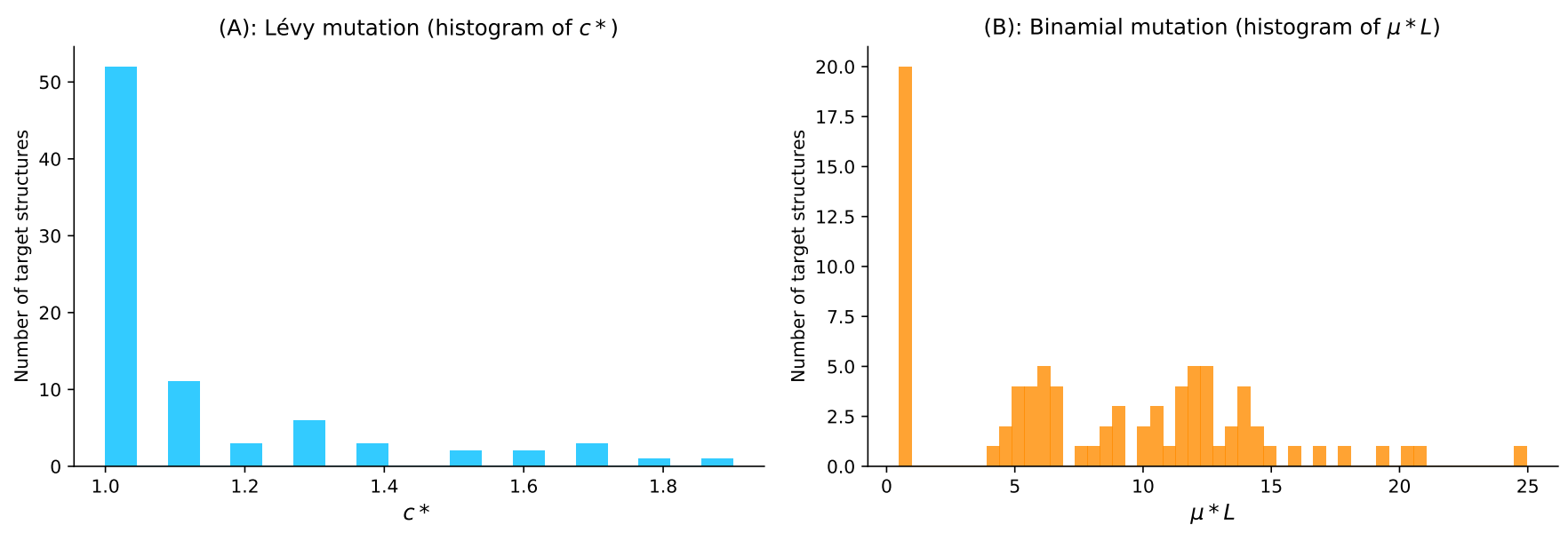

Fig. 3. Parameter tuning for both binomial and Lévy mutation schemes.(A) Lévy mutation parameter tuning. Histogram of best exponent parameter $\left(c^{*}\right)$ for a set of 81 target structures with different pseudoknot patterns and various lengths. The most frequent best exponent value is 1 . (B) Binomial parameter tuning. Histogram of best mutation rate $\left(\mu^{*}\right)$ for the same set of 81 target structures with different pseudoknots and various lengths. The most frequent best parameter is the low mutation rate $(\approx 1 / L)$. For some structures, the best mutation rate is the high one for different lengths as well.

Throughout this paper, local mutation will refer to binomial distributed mutation with parameter $\mu \approx 1 / L$ or to one-point mutation.

New feature. We provide an updated version of aRNAque supporting pseudoknotted RNA target structures. In addition to the support for pseudoknots, we provide an updated mutation mode based on a Zipf distribution. We present the mutation algorithm in Algorithm 1.

\section{Parameter analysis and benchmark}

Here we analyse mutation parameters and compare local and Lévy mutation modes.

Benchmark data. To compare our new version of aRNAque with existing tools in the literature, we used the PseudoBase++ benchmark datasets for pseudoknotted target structures and the Eterna100 dataset for pseudoknot-free target structures.

The PseudoBase++ is a set of 265 pseudoknotted RNA structures used to benchmark Modena. It was initially 304 RNA secondary structures, but we excluded 37 because they had non-canonical base pairs. We then grouped the structures into four pseudoknot motifs (Figure 2): 209 hairpin pseudoknots (H), 29 bulge pseudoknots (B), 8 complex hairpin pseudoknots $(\mathrm{cH})$ and 4 kissing hairpin pseudoknots $(\mathrm{K})$.

The Eterna100 dataset (34) is available in two versions and both contain a set of 100 target structures extracted from the EteRNA puzzle game and classified by their degree of difficulty. The Eterna100-V1 was initially designed using ViennaRNA 1.8.5, which relies on Turner1999 energy parameters (35). Out of the 100 target secondary structures, 19 turned out to be unsolvable using the recent version of ViennaRNA (Version 2.14). Subsequently, an Eterna100-V2 (34) was released in which the 19 targets were slightly modified to be solvable using ViennaRNA 2.14 .
Methodology. The best mutation parameters obtained for both binomial and Lévy mutation modes are used to benchmark and compare the results on the entire datasets of RNA structures (265 from PseudoBase++ and 100 from EteRNA100). First, for each of the 365 target structures $\sigma^{*}$ in the datasets, 20 sequences were designed. To measure the performance of each tool, each designed sequence $s$ is folded into a secondary structure $\sigma$ and the similarities between $\sigma$ and $\sigma^{*}$ are computed using the base pair distance. Second, for each of the Eterna 100 target structures and a maximum of 5000 generations (i.e. 50,000 evaluations), 5 to 20 runs were launched independently, which results in at least 5 designed sequences per target. We define success rate simply as the number of successfully designed targets. A target is considered successfully designed when at least one of the designed sequence folds into the target structure (i.e. the Hamming distance between the target structure and the MFE structure is 0 ).

Folding tools. Two tools for pseudoknotted RNA folding are considered in this work: HotKnots and IPknot. For pseudoknot-free RNA folding, we used RNAfold. For the mutation parameter analysis presented here, we used IPknot, and both HotKnots and IPknot for pseudoknotted targets. Furthermore, we considered pkiss, a well know tool for K-type pseudoknot prediction, but since the PseudoBase++ dataset contains just 5 K-type pseudoknotted structures and pKiss has higher time complexity $\left(O\left(n^{6}\right)\right)$, we did not find it efficient for the benchmark we performed.

Mutation parameters tuning. One of the main challenges for an evolutionary algorithm is to find optimum parameters such as mutation rate, population size and selection function. We used 81 pseudoknotted targets with lengths from 25 to 181 nucleotides for the mutation parameter analysis. We set the maximum number of generations to 200 and the population size to 100 . The best mutation parameters $\left(c^{*}\right.$ for Levy and 

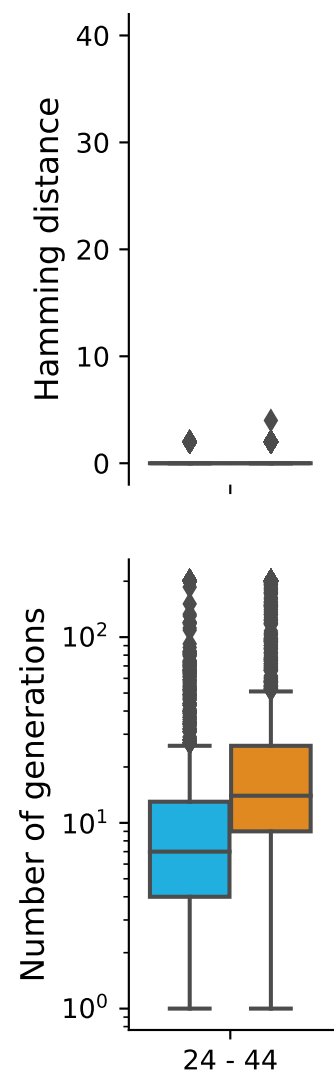
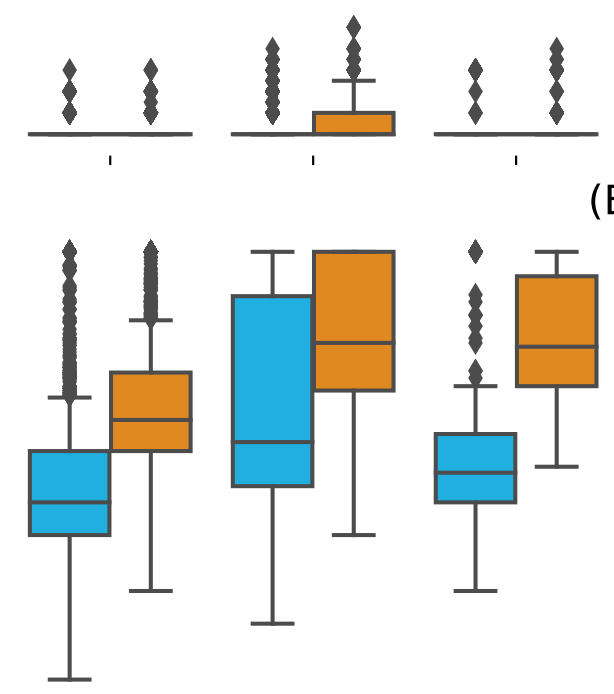

(A)

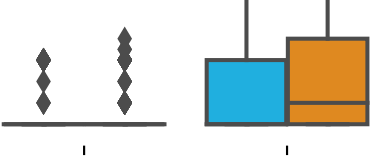

(B)
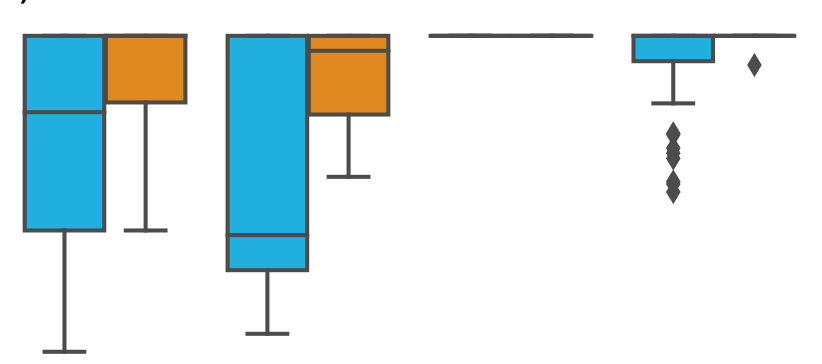

Lévy mutation Local mutation
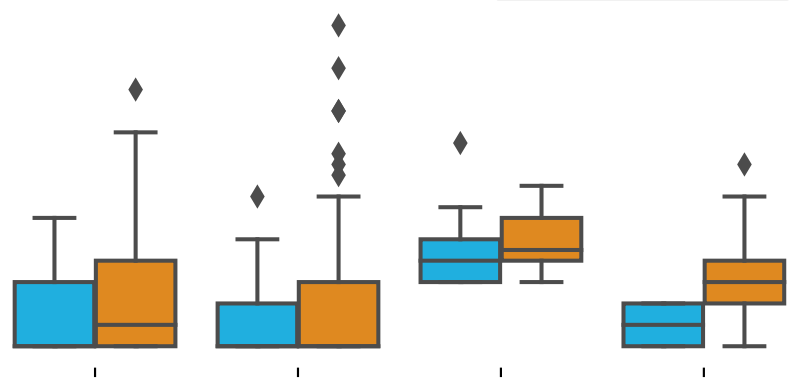

$64-84$

84 - 104

$104-124$

$124-144$

$144-164$

$164-184$

Fig. 4. Lévy mutation mode vs local mutation (one-point mutation). (A) Hamming distance distributions vs. target structure lengths. (B) Number of generations distributions for different length groups. In both (A) and (B), lower values indicate better performance. The target structures are solvable in less than 100 generations for both mutation schemes and most length groups. Still, the difference in the number of generations gets more significant as the target lengths increase, except for the two last length groups for which both mutation schemes mostly failed. The highest difference in terms of median number of generations is 150 for target lengths in the range [124 - 144] (respectively $123,49,46,16,7,0,0$ for the length ranges [84 - 104], [64 - 84], [104-124], [44-64], [24 - 44], [144-164], [164 - 184]). Averaging over all length groups, the median number of generations difference between the Levy mutation and the one point mutation is 48 generations.

$\mu^{*}$ for Binomial) are those that have the lowest median number of generations.

- Binomial mutation: From Figure 1B, the critical range was identified to be from 0 to 0.2 and as $\mu$ becomes greater than 0.1 , the success rate decreases and the average number of generations increases. For each of the 80 target structures with pseudoknots, 20 sequences were designed for $\mu \in[0,0.2]$ with a step size of $1 / L$. Figure $3 \mathrm{~B}$ shows the histogram of the best mutation rate found for each target structure. Two main regimes are apparent: one where the best mutation rate is very low mutation rate $(\approx 1 / L)$ and another where the high mutation rate is optimal.

- Lévy mutation: From Figure 1C, the critical range of $c$ was identified to be $[1,2]$. For $c \in[1,2]$ and a step size of 0.1 , an optimum exponent parameter $c^{*}$ was investigated for all the 80 target structures. Figure $3 \mathrm{~A}$ shows the histogram of $c^{*}$. Contrary to binomial mutation, the optimum exponent parameter does not vary too much $\left(\forall \sigma, c^{*} \approx 1\right)$.

Figure 3A shows that when using a Lévy mutation, the opti- mal mutation rate is approximately the same for most target structures. In contrast, the optimum binomial mutation rate parameter $\mu^{*}$ mostly varies with different targets. In Figures $1 \mathrm{~B}$ and $1 \mathrm{C}$, although both mutation modes have approximately the same success rates ( $88 \%$ for the Lévy over 100 runs and $\approx 92 \%$ for the binomial over 50 runs), the Lévy flight mutation scheme is more robust to different targets. Moreover, the median number of generations for the Lévy mutation is lower ( 54 for the Lévy and 92 for the binomial mutation mode), thus enhancing efficiency.

\section{Results}

We first compared the performance of aRNAque using Lévy mutations to the previous version with local mutations (binomial number of point-mutations with $\mu \approx \frac{1}{L}$ ). Secondly, we compared aRNAque to the existing pseudoknotted RNA inverse folding tool ant aRNA using two folding tools: HotKnots and IPknot. We used the PseudoBase++ dataset for both benchmarks.

Performance on PseudoBase+t: Levy mutation vs. local mutation. Figure 4 shows box plots for the base pair 
(A) IPknot

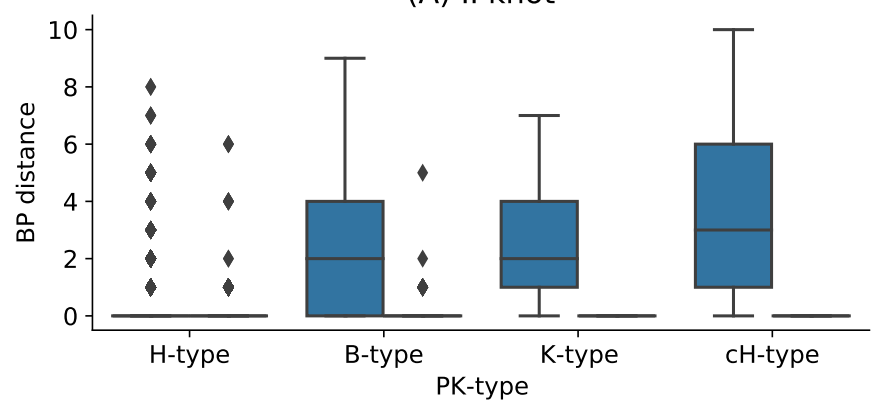

(C) IPknot

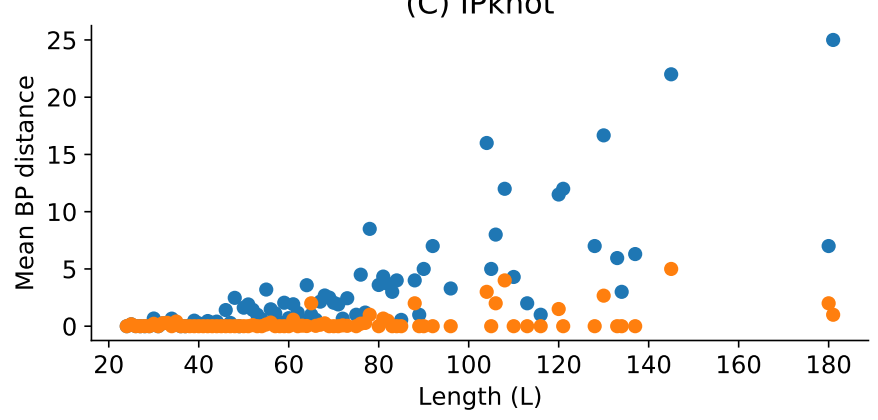

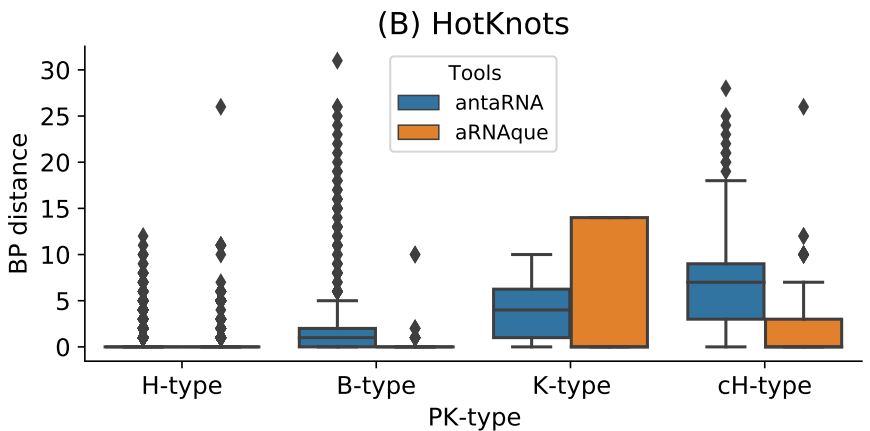

(D) HotKnots

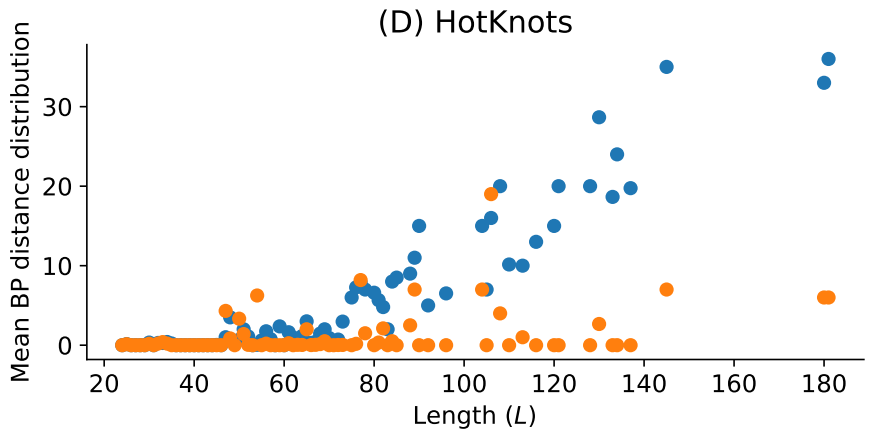

Fig. 5. aRNAque vs antaRNA on PseudoBase++ dataset using both IPknot and HotKnots. Lower values imply better performance. (A, B) Base pair distance distributions of the designed sequences to the target structure for different pseudoknot types. (C,D) Mean base pair distance against target lengths.

distance (Hamming distance) and the number of generations for increasing target lengths under our two mutation schemes: binomial at low mutation rate (or one point mutation) and the Lévy mutation. For each pseudoknotted RNA target structure in the PseudoBase++ dataset, we designed 20 sequences. The results show that using the Lévy mutation instead of a local mutation scheme can significantly increase the performance of aRNAque. The gain was less significant in terms of designed sequences quality (base pair distance distributions, with a $t$-value $\approx-1.04$ and $p$-value $\approx 0.16$ ) but more significant in terms of the average minimum number of generations needed for successful matches to target structures (with a $t$-value $\approx-3.6$ and $p$-value $\approx 0.0004$ ). This result demonstrates a substantial gain in computational time when using a Lévy mutation scheme instead of a purely local mutation.

Performance on PseudoBase+t: aRNAque vs. antaRNA. We also compared the sequences designed using aRNAque (with the Lévy mutation scheme) to those produced by antaRNA. Figures 5A and 5C show the base pair distance distribution for each category of pseudoknotted target structure and the mean of the base pair distance plotted against the length of the target secondary structures. For antaRNA, and when using IPknot as a folding tool, finding sequences that fold into the target becomes increasingly difficult with pseudoknot complexity (median base-pair distance distribution increases). On the other hand, aRNAque's performance improves as pseudoknot complexity increases (e.g. the mean base-distance decreases with the pseudoknot complexity). In sum, as target length increases, the performance of antaRNA (local search) is considerably degraded , while aRNAque (Lévy flight search) stays almost constant.
A second benchmark using HotKnots as a folding tool was performed on the same dataset. For both aRNAque and antaRNA, the more complex the pseudoknot motifs, the worse is the tool performance (median of the basepair distance distribution increases). Figures 5B and 5D show the base pair distance distributions with respect to the pseudoknot motifs for both aRNAque and antaRNA. Even though both performances degrade as target length increases, aRNAque (Lévy flight evolutionary search) performance remains almost constant for all the target lengths greater than 60 .

Performance on Eterna100 dataset. Finally, we performed a third benchmark on the Eterna100 datasets. First, on the Eterna100-V1 dataset, the Lévy flight version of aRNAque successfully designed $89 \%$ of the targets and the one-point mutation (local mutation) version achieved $91 \%$ of success, suggesting that for some target structures, local mutation can outperform the Lévy mutation scheme. Combining the two datasets, aRNAque solved in total $92 \%$ of the targets of Eterna100-V1 (see also (23)).

When analysing the performance of Lévy flight for low and high base pair densities separately, the median number of generations of high base pair density targets was lower than the one with low base-pair density ( 8 generations for high density and 18 for the low base pairs density targets). The same observation was drawn for the success rate. For the low base-pair density targets, the Lévy flight achieved $87 \%$ (49/56) success whereas, for the high base-pair density, it achieved $91 \%$ (40/44). The same analysis can be done when comparing the one-point mutation results for the high-density targets to the Lévy flight mutation. The median number of 
(A)

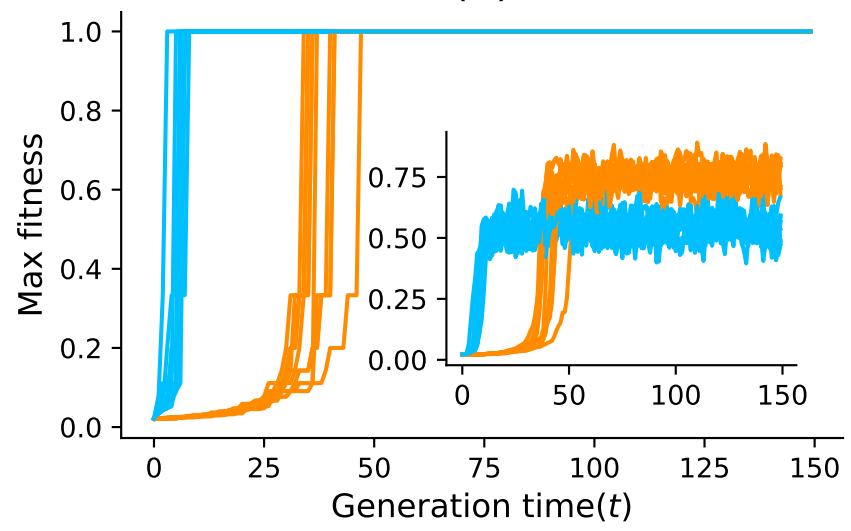

(C)

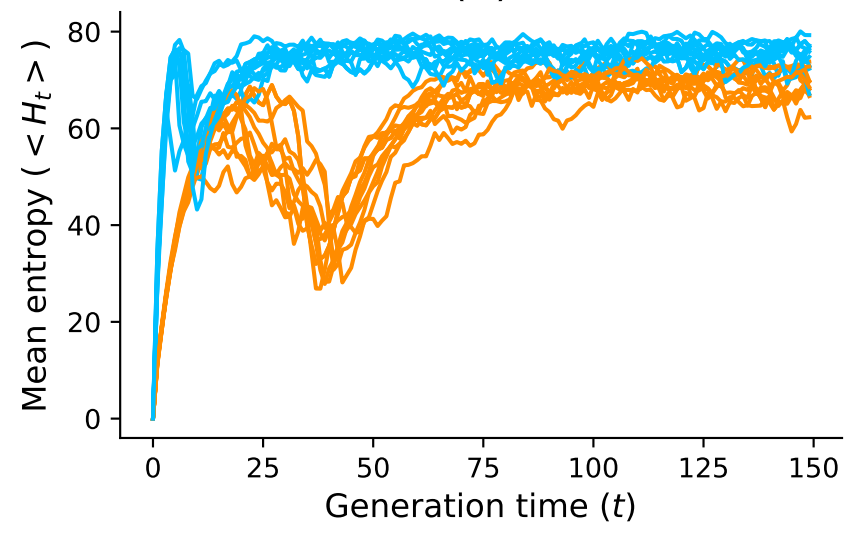

(B)

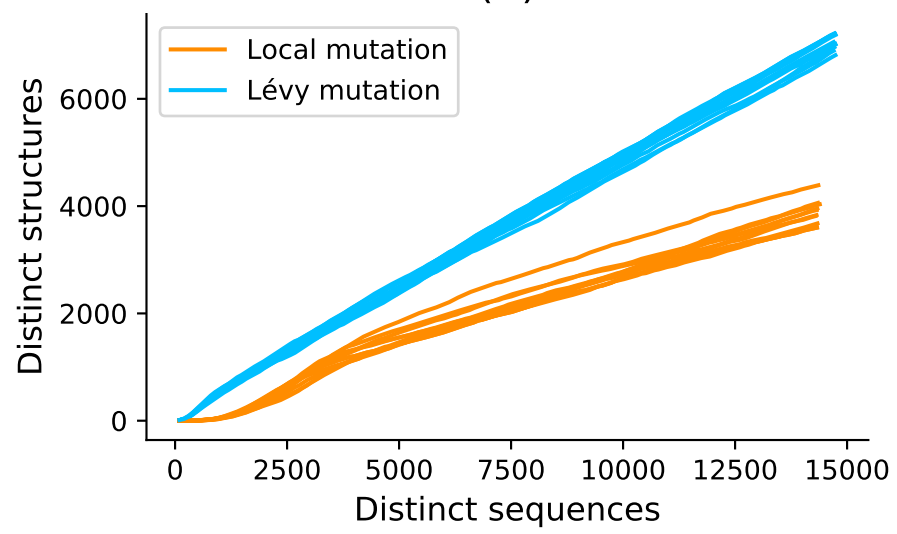

(D)

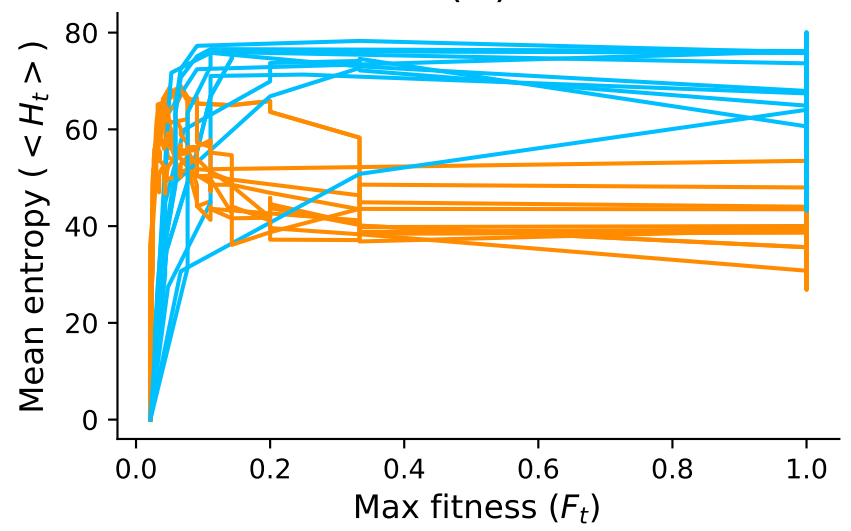

Fig. 6. Lévy mutation vs one-point mutation: diversity analysis. For the Eterna 100 target structure [CloudBeta] 5 Adjacent Stack Multi-Branch Loop, ten independent runs were performed in which a minimum of 10 sequences were designed per run. (A) Max fitness and mean fitness (inset) over time. (B) Distinct sequences vs. Distinct structures over time. (C) Mean Shannon entropy of the population sequences over time for both binomial and Lévy mutation. (D) The max fitness plotted against the entropy over time.

generations for the low-density targets when using a onepoint mutation operator was 34 (respectively 24 for the high base pair density targets) (see Figure 7A).

A new benchmark was performed on Eterna100-V2 with aRNAque achieving a $93 \%$ success rate. Compared to recently reported benchmark results (34), aRNAque achieved similar performance to NEMO on Eterna-V2: one target was unsolved by all existing tools and one target solved only by NEMO remained unsolved by aRNAque.

\section{Discussion}

In this work, we provide an updated version of aRNAque implementing a Lévy flight mutation scheme that supports pseudoknottted RNA secondary structures. A Lévy mutation scheme offers a compromise between exploration at different scales (mostly local search combined with rare big jumps). Such a scheme significantly improves the number of evaluations needed to hit the target structure, while better avoiding getting trapped in local optima. The benefit of a Lévy flight over a purely local (binomial with $\mu<<1$ or a single point mutation) mutation search allowed us to explore RNA sequence space at all scales. Such a heavy tailed distribution in the number of point mutations permitted the design of more diversified sequences and reduced the number of evaluations of the evolutionary algorithm implemented in
aRNAque. The main advantage of using a Lévy flight over local search is a reduction in the number of generations required to reach a target. This is because the infrequent occurrence of a high number of mutations allow a diverse set of sequences among early generations, without the loss of robust local search. One consequence is a rapid increase in the population mean fitness over time and a rapid convergence to the target of the maximally fit sequence. To illustrate that advantage, we ran aRNAque starting from an initial population of unfolded sequences, both for a "one point mutation" and "Lévy mutation".

Figures $6 \mathrm{~A}$ and $6 \mathrm{~B}$ show respectively the max/mean fitness over time and the number of distinct structures discovered over time plotted against the number of distinct sequences. When using a Lévy mutation scheme, the mean fitness increases faster in the beginning but stays lower than that using local mutations. Later in the optimisation, a big jump or high mutation on the RNA sequences produces structures with fewer similarities and, by consequence, worse fitness. In the $(5-10)^{t h}$ generation, sequences folding into the target are already present in the Lévy flight population, but only at the $30^{t h}$ generation are similar sequences present in the local search population. The Lévy flight also allows exploration of both the structure and sequence spaces, providing a higher diversity of structures for any given set of sequences 

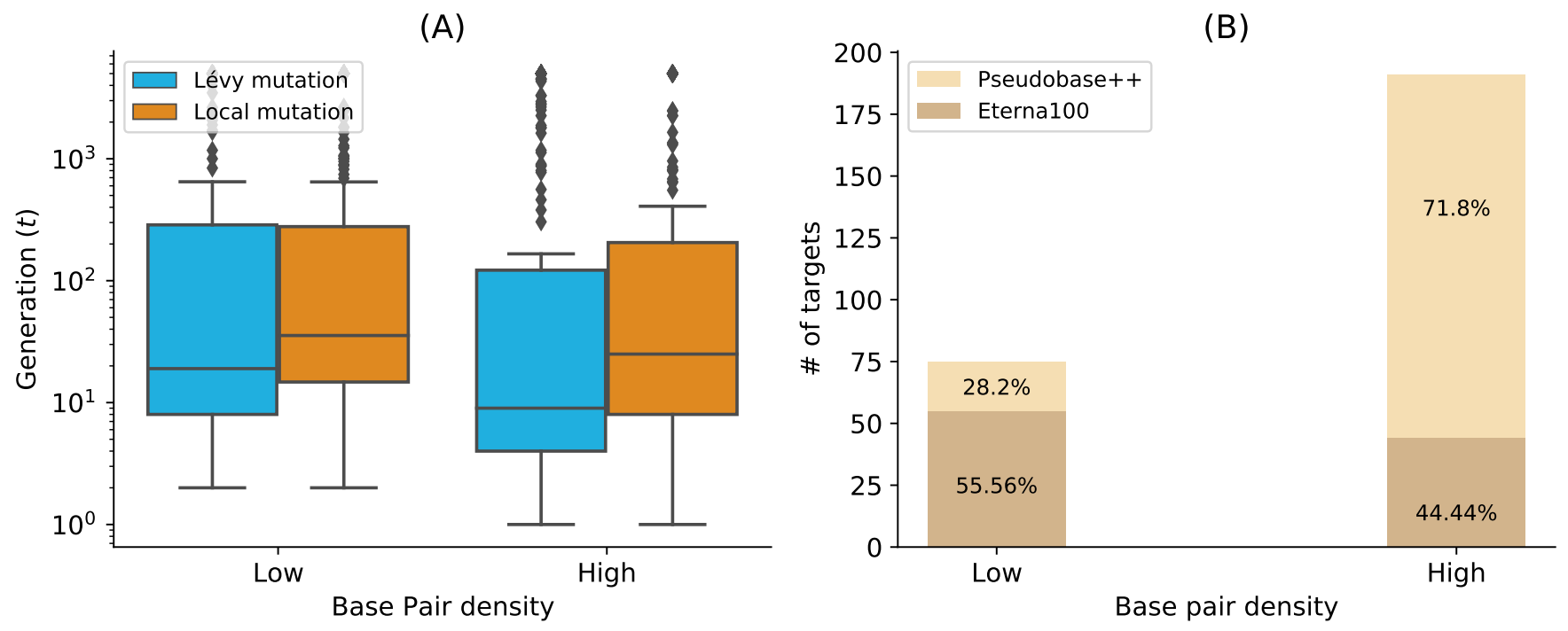

Fig. 7. Lévy mutation vs. Local mutation: performance analysis with respect to the base-pair density. The higher the base-pair density is, the more useful the Lévy mutation scheme to speed up the optimization EA. (A) Distributions of number of generations for the low and high base-pair density targets of the Et erna 100 dataset. (B) Percentages of targets with low and high base-pair density for the Eterna100 and PseudBase++

(Figure 6B). Using the mean entropy of structures as an alternate measure of diversity, we see in Figures 6C and 6D how a Lévy flight achieves high diversity early in implementation, and maintains a higher diversity over all generations than a local search algorithm. Although the mutation parameters $P_{C}$ and $P_{N}$ influence the absolute diversity of the designed sequences, the Lévy flight always tends to achieve a higher relative diversity than local search, all else being equal.

We argue that the improved performance of the Lévy Flight over local search in target RNA structures is due to the high base pair density of pseudoknotted structures. Given that pseudoknots present a high density of interactions, there are dramatic increases in possible incorrect folds and thus increasing risk of becoming trapped near local optima (36). Large numbers of mutations in paired positions, as implied by a heavy tailed distribution, are necessary to explore radically different solutions.

To illustrate that Lévy Flight performance was due to base pair density, we clustered the benchmark datasets into two classes: one cluster for target structures with low base pair density (density $\leq 0.5$ ) and a second cluster for structures with high base pair density (density $>0.5$ ). Figure 7B shows the number of target sequences available in each low and high density category. The number of targets available in each category are colored according to the percentage of pseudoknotfree targets (Eterna100-V1) vs. targets with pseudoknots (Pseudobase++), showing that pseudoknots are strongly associated with high base pair densities: $71 \%$ of the pseudoknotted target structures have a high base pair density. In contrast, the Eterna100 dataset without psuedoknots has somewhat higher representation at low base pair density. If it is true that improved Lévy Flight performance is indeed tied to base pair density, it is possible that similar heavytailed mutation schemes could offer a scalable solution to even more complex inverse folding problems.

Although we believe that Lévy flight-type search algorithms offer a valuable alternative to local search, we emphasise that its enhanced performance over say antaRNA is partially influenced by the specific capabilities of existing folding tools. Their limitations may account for the degradation of these tools as the pseudoknot motifs get increasingly complex. Another possible limitation is the fact that most target structures were relatively easy to solve (in less than 100 generations), which possibly allowed local search to perform better than Lévy search in some cases. Further research on more challenging target structures will improve our understanding of which conditions favour local $v s$. Lévy search.

\section{Conclusion}

Our results show general and significant improvements in the design of RNA secondary structures compared to the standard evolutionary algorithm mutation scheme with a mutation parameter $\approx 1 / L$, where $L$ is the sequence solution length. Not only does Lévy flight mutations lead to greater diversity of RNA sequence solutions, but it also reduces the evolutionary algorithm's number of evaluations, thus improving computing time.

\section{Availability}

The implementation in python 3.7 of aRNAque and the benchmark data used in this manuscript are available at https://github.com/strevol-mpi-mis/ aRNAque. We also provide the scripts used for the figures and the designed sequences analysis.

\section{Competing interests}

The authors declare that they have no competing interests.

\section{Author's contributions}

Nono S. C. Merleau contributed in the investigation, software coding, visualization, writing - original draft, and writing - 
bioRxiv preprint doi: https://doi.org/10.1101/2022.01.17.476593; this version posted January 20, 2022. The copyright holder for this preprint (which was not certified by peer review) is the author/funder, who has granted bioRxiv a license to display the preprint in perpetuity. It is made available under aCC-BY-NC-ND 4.0 International license.

review \& editing. Matteo Smerlak contributed in the funding acquisition, investigation, methodology, Supervision, validation and the writing - review \& editing

\section{Funding}

Funding for this work was provided by the Alexander von Humboldt Foundation in the framework of the Sofja Kovalevskaja Award endowed by the German Federal Ministry of Education and Research, and by the Human Science Frontier Program Organization through a Young Investigator Award.

\section{Consent for publication}

All authors agree with its contents and with this submission.

\section{Acknowledgements}

We thank the Structure of Evolution Group at the Max Planck Institute for Mathematics in the Sciences, and especially Vincent Messow, Opuu Vaitea and Ian Hatton for useful discussions and insightful comments. The Alexander von Humboldt Foundation provided funding for this work in the framework of the Sofja Kovalevskaja Award endowed by the German Federal Ministry of Education.

\section{References}

1. Nadrian C Seeman, John M Rosenberg, FL Suddath, Jung Ja Park Kim, and Alexander Rich. RNA double-helical fragments at atomic resolution: I. The crystal and molecular structure of sodium adenylyl- $3^{\prime}, 5^{\prime}$-uridine hexahydrate. Journal of molecular biology, 104 (1):109-144, 1976.

2. John M Rosenberg, Nadrian C Seeman, Roberta O Day, and Alexander Rich. RNA doublehelical fragments at atomic resolution: II. The crystal structure of sodium guanylyl- $3^{\prime}, 5^{\prime}$ cytidine nonahydrate. Journal of molecular biology, 104(1):145-167, 1976.

3. Eric Westhof and Valérie Fritsch. RNA folding: beyond Watson-Crick pairs. Structure, 8(3): R55-R65, 2000. ISSN 0969-2126. doi: https://doi.org/10.1016/S0969-2126(00)00112-X.

4. Michael S Goldberg, Deyin Xing, Yin Ren, Sandra Orsulic, Sangeeta N Bhatia, and Phillip A Sharp. Nanoparticle-mediated delivery of siRNA targeting parp1 extends survival of mice bearing tumors derived from BRCA1-deficient ovarian cancer cells. Proceedings of the National Academy of Sciences, 108(2):745-750, 2011.

5. Chenhui Hao, Xiang Li, Cheng Tian, Wen Jiang, Guansong Wang, and Chengde Mao. Construction of RNA nanocages by re-engineering the packaging RNA of phi29 bacteriophage. Nature communications, 5:3890, 2014.

6. Maung Nyan Win and Christina D Smolke. Higher-order cellular information processing with synthetic RNA devices. Science, 322(5900):456-460, 2008.

7. Ronny Lorenz, Stephan H Bernhart, Christian Höner Zu Siederdissen, Hakim Tafer, Christoph Flamm, Peter F Stadler, and Ivo L Hofacker. ViennaRNA package 2.0. Algorithms for molecular biology, 6(1):26, 2011.

8. Vaitea Opuu, Nono SC Merleau, and Matteo Smerlak. RAFFT: Efficient prediction of RNA folding pathways using the fast fourier transform. bioRxiv, 2021.

9. Elena Rivas and Sean R. Eddy. A dynamic programming algorithm for RNA structure prediction including pseudoknots. Journal of Molecular Biology, 285(5):2053-2068, 1999. ISSN 0022-2836. doi: https://doi.org/10.1006/jmbi.1998.2436.

10. Kengo Sato, Yuki Kato, Michiaki Hamada, Tatsuya Akutsu, and Kiyoshi Asai. IPknot: fast and accurate prediction of RNA secondary structures with pseudoknots using integer programming. Bioinformatics, 27(13):i85-i93, 2011.

11. Jihong Ren, Baharak Rastegari, Anne Condon, and Holger H Hoos. HotKnots: heuristic prediction of RNA secondary structures including pseudoknots. RNA, 11(10):1494-1504, 2005.

12. Ali Esmaili-Taheri and Mohammad Ganjtabesh. ERD: a fast and reliable tool for RNA design including constraints. BMC bioinformatics, 16(1):20, 2015.

13. N Dromi, A Avihoo, and D Barash. Reconstruction of natural RNA sequences from RNA shape, thermodynamic stability, mutational robustness, and linguistic complexity by evolutionary computation. Journal of Biomolecular Structure and Dynamics, 26(1):147-161, 2008.

14. Ali Esmaili-Taheri, Mohammad Ganjtabesh, and Morteza Mohammad-Noori. Evolutionary solution for the RNA design problem. Bioinformatics, 30(9):1250-1258, 2014.

15. Akito Taneda. Modena: a multi-objective RNA inverse folding. Advances and applications in bioinformatics and chemistry: $A A B C, 4: 1,2011$.

16. Jade Shi, Rhiju Das, and Vijay S Pande. SentRNA: Improving computational RNA design by incorporating a prior of human design strategies. arXiv preprint arXiv:1803.03146, 2018.
17. Alexander Churkin, Matan Drory Retwitzer, Vladimir Reinharz, Yann Ponty, Jérôme Waldispühl, and Danny Barash. Design of RNAs: comparing programs for inverse RNA folding. Briefings in bioinformatics, 19(2):350-358, 2017.

18. Juan Antonio Garcia-Martin, Peter Clote, and Ivan Dotu. RNAiFOLD: a constraint programming algorithm for RNA inverse folding and molecular design. Journal of bioinformatics and computational biology, 11(02):1350001, 2013.

19. Hua-Ting Yao, Jérôme Waldispühl, Yann Ponty, and Sebastian Will. Taming disruptive base pairs to reconcile positive and negative structural design of RNA. In RECOMB 2021-25th international conference on research in computational molecular biology, 2021.

20. Robert Kleinkauf, Martin Mann, and Rolf Backofen. antaRNA: ant colony-based RNA sequence design. Bioinformatics, 31(19):3114-3121, 2015.

21. Akito Taneda. Multi-Objective Genetic Genetic for Pseudoknotted RNA Sequence Design. Frontiers in Genetics, 3:36, 2012. ISSN 1664-8021. doi: 10.3389/fgene.2012.00036.

22. James ZM Gao, Linda YM Li, and Christian M Reidys. Inverse folding of RNA pseudoknot structures. Algorithms for Molecular Biology, 5(1):1-19, 2010.

23. Nono SC Merleau and Matteo Smerlak. A simple evolutionary algorithm guided by local mutations for an efficient RNA design. In Proceedings of the Genetic and Evolutionary Computation Conference, pages 1027-1034, 2021.

24. B Mandelbrot. Certain speculative prices (1963). The Journal of Business, 45(4):542-543, 1972.

25. Micheal F Shlesinger, George M Zaslavsky, and Uriel Frisch. Lévy flights and related topics in physics. Springer, 1995.

26. Gandhimohan M Viswanathan, EP Raposo, and MGE Da Luz. Lévy flights and superdiffusion in the context of biological encounters and random searches. Physics of Life Reviews, 5(3):133-150, 2008.

27. Anis Farhan Kamaruzaman, Azlan Mohd Zain, Suhaila Mohamed Yusuf, and Amirmudin Udin. Lévy flight algorithm for optimization problems-a literature review. In Applied Mechanics and Materials, volume 421, pages 496-501. Trans Tech Publ, 2013.

28. Andy M Reynolds. Current status and future directions of lévy walk research. Biology open, 7(1):bio030106, 2018.

29. Mark EJ Newman. Power laws, Pareto distributions and Zipf's law. Contemporary physics, 46(5):323-351, 2005.

30. Christian Darabos, Mario Giacobini, Ting Hu, and Jason H. Moore. Lévy-Flight Genetic Programming: Towards a New Mutation Paradigm. In Mario Giacobini, Leonardo Vanneschi, and William S. Bush, editors, Evolutionary Computation, Machine Learning and Data Mining in Bioinformatics, pages 38-49, Berlin, Heidelberg, 2012. Springer Berlin Heidelberg. ISBN 978-3-642-29066-4.

31. Vishnu Prakash Sharma, Harji Ram Choudhary, Sandeep Kumar, and Vikas Choudhary. A modified DE: Population or generation based levy flight differential evolution (PGLFDE). In 2015 International Conference on Futuristic Trends on Computational Analysis and Knowledge Management (ABLAZE), pages 704-710. IEEE, 2015.

32. Michela Taufer, Abel Licon, Roberto Araiza, David Mireles, FHD Van Batenburg, Alexander P Gultyaev, and Ming-Ying Leung. Pseudobase++: an extension of pseudobase for easy searching, formatting and visualization of pseudoknots. Nucleic acids research, 37 (suppl 1):D127-D135, 2009.

33. Jeff Anderson-Lee, Eli Fisker, Vineet Kosaraju, Michelle Wu, Justin Kong, Jeehyung Lee, Minjae Lee, Mathew Zada, Adrien Treuille, and Rhiju Das. Principles for predicting rna secondary structure design difficulty. Journal of molecular biology, 428(5):748-757, 2016.

34. Rohan V. Koodli, Boris Rudolfs, Hannah K. Wayment-Steele, Eterna Structure Designers, and Rhiju Das. Redesigning the EteRNA100 for the Vienna 2 folding engine. bioRxiv, 2021. doi: $10.1101 / 2021.08 .26 .457839$.

35. Douglas $\mathrm{H}$. Turner and David H. Mathews. NNDB: the nearest neighbor parameter database for predicting stability of nucleic acid secondary structure. Nucleic Acids Research, 38 (suppl1):D280-D282, 2009.

36. Christine E Hajdin, Stanislav Bellaousov, Wayne Huggins, Christopher W Leonard, David H Mathews, and Kevin M Weeks. Accurate SHAPE-directed RNA secondary structure modeling, including pseudoknots. Proceedings of the National Academy of Sciences, 110(14): 5498-5503, 2013. 